T. Matsuzawa

Nagoya Math. J.

Vol. 108 (1987), 53-66

\title{
A CALCULUS APPROACH TO HYPERFUNCTIONS I
}

\author{
TADATO MATSUZAWA
}

To the memory of C. Goulaouic

\section{Introduction}

In this paper, we shall give a new characterization of hyperfunctions without algebraic method and apply to give simpler proofs to problems discussed in [3], Chapter 9. In [3], the spaces of hyperfunctions $A^{\prime}(K)$ with compact support in $K \subset R^{n}(n \geqq 1)$ is considered as the dual of the space $A(K)$ of functions which are real analytic near $K$. Each element $u$ of $A^{\prime}(K)$ is characterized as a density of a double layer potential in $R^{n} \times R$.

We shall first give a simpler characterization of the element $u$ of $A^{\prime}(K)$ in Theorem 1.2. We will consider $u \in A^{\prime}(K)$ as an initial value of a unique solution of the heat equation

$$
(\partial / \partial t-\Delta) U(x, t)=0 \quad \text { in } R_{+}^{n+1}=R^{n} \times R_{+} .
$$

The regularity of $u=U(\cdot, 0)$ is described by the asymptotic behavior of $U$ as $t \rightarrow 0$. In fact, we characterize in Theorem 1.2 the asymptotic behavior so that $u \in A^{\prime}(K)$. We will see Schwartz and ultradistributions are characterized by the asymptotic behavior of $U$ at the same time. The advantage of our approach is to unify the theory of distributions and hyperfunctions as well as to simplify proofs of important results. For example, based on Theorem 1.2, we significantly simplify the proof of Paley-Wiener-Schwartz theorem for hyperfunctions as well as Schwartz distributions in Section 2. We discuss in Section 3 hypoellipticity of a pseudodifferential equation

$$
a(x, D) u=f \quad \text { in } \Omega \subset R^{n},
$$

where $u \in A^{\prime}(K), K \Subset \Omega, f$ is a hyperfunction on $\Omega$ and $a(x, D)$ is analytic pseudodifferential operator considered in [1], [9] and [10]. Analytic and

Received March 17, 1986. 
$C^{\infty}$-hypoellpticity of the above equation will be proved in Theorem 3.2. Our theory is based just on calculus. We never appeal to cohomology, sheaves nor theory of several complex variables. The other problems concerning hyperfunctions will be considered in a forthcoming paper.

The author expresses his profound gratitude to Y. Morimoto and Y. Giga for valuable discussions during the preparation of this paper.

\section{§1. Hyperfunctions}

Let $K$ be a compact set of $R^{n}$ whose point is denoted by $x=\left(x_{1}, \cdots, x_{n}\right)$. We use general notations such as $|\alpha|=\alpha_{1}+\cdots+\alpha_{n}$ for a multi-index $\alpha=\left(\alpha_{1}, \cdots, \alpha_{n}\right)$ and $D^{\alpha}=D_{1}^{\alpha_{1}} \cdots D_{n}^{\alpha_{n}}, D_{j}=1 / i\left(\partial / \partial x_{j}\right), j=1, \cdots, n$, etc. Let $A(K)$ be the space of all real analytic functions in some neighborhood of $K$. That is, if $\phi \in A(K) \phi$ is a $C^{\infty}$ function in a neighborhood of $K$ and there are positive constants $C$ and $h$ such that

$$
\sup _{\substack{x \in K \\ \alpha}} \frac{\left|D^{\alpha} \phi(x)\right|}{h^{|\alpha|} \alpha !} \leqq C .
$$

Definition 1.1. We say $\phi_{j} \rightarrow 0$ in $A(K)$ as $j \rightarrow \infty$ if there is a constant $h>0$ such that

$$
\sup _{x \in K} \frac{\left|D^{\alpha} \phi_{j}(x)\right|}{h^{|\alpha|} \alpha !} \longrightarrow 0 \quad \text { as } j \longrightarrow \infty .
$$

Definition 1.2 (cf. [3] and [8]). We denote by $A^{\prime}(K)$ the strong dual space of $A(K)$ and call its elements analytic functionals carried by $K$. We denote by $A$ the space of entire analytic functions in $C^{n}$.

THEOREM 1.1. Let $u \in A^{\prime}(K)$ then for any $h>0$ there exists a constant $C=C(h, u)$ such that

$$
|u(\phi)| \leqq C \sup _{x \in K} \frac{\left|D^{\alpha} \phi(x)\right|}{h^{|\alpha|} \alpha !}, \quad \phi \in A .
$$

This is equivalent to the condition such that for every complex neighborhood $\omega$ of $K$

$$
|u(\phi)| \leqq C_{\omega} \sup _{\omega}|\phi|, \quad \phi \in A .
$$

Proof. The equivalence of (1.3) and (1.4) follows easily from Cauchy's integral formula and Taylor expansion of analytic functions. So we shall prove (1.4) for $u \in A^{\prime}(K)$. We suppose that the estimate (1.4) does not hold 
for some $u \in A^{\prime}(K)$. Then there must be a complex neighborhood $\omega$ of $K$ such that for any integer $j$ there is a function $\phi_{j}$ satisfying

$$
\left|u\left(\phi_{j}\right)\right| \geqq j \sup _{\omega}\left|\phi_{j}\right| \text {. }
$$

We may suppose $\sup _{\omega}\left|\phi_{j}\right|=1, j=1,2, \cdots$. Then we have

$$
\phi_{j} / j \longrightarrow 0 \quad \text { in } A(K) \text { as } j \longrightarrow \infty \text {. }
$$

On the other hand, we have $\left|u\left(\phi_{j} \mid j\right)\right| \geqq 1, j=1,2, \cdots$, which contradicts with the continuity of $u$.

Proposition 1.1 (cf. [3] Proposition 9.1.2.). For every $\phi \in A(K)$ let

$$
\phi_{t}(x)=(4 \pi t)^{-n / 2} \int_{R^{n}} \exp \left(-(x-y)^{2} / 4 t\right) \chi(y) \phi(y) d y,
$$

where $\chi \in C_{0}^{\infty}\left(R^{n}\right)$ such that $\chi=1$ in a neighborhood of $K$. Then $\phi_{t}(x) \rightarrow$ $\phi(x)$ in $A(K)$ as $t \rightarrow 0_{+}$.

By virtue of this proposition, we can see that (1.3) is equivalent to

$$
|u(\phi)| \leqq C \sup _{x \in K} \frac{\left|D^{\alpha} \phi(x)\right|}{h^{|\alpha|} \alpha !}, \quad \phi \in A(K)
$$

with the same constant $C$ in (1.3).

Now denote by $E(x, t)$ the $n$-dimensional heat kernel:

$$
E(x, t)=\left\{\begin{array}{cl}
(4 \pi t)^{-n / 2} \exp \left(-|x|^{2} / 4 t\right) & t>0 \\
0 & t<0
\end{array}\right.
$$

and set

$$
U(x, t)=u_{y}(E(x-y, t)), \quad t>0, x \in R^{n} .
$$

TheOREM 1.2. Let $u \in A^{\prime}(K)$ then $U(x, t) \in C^{\infty}\left(R_{+}^{n+1}\right), R_{+}^{n+1}=\{(x, t) ; x \in$ $\left.R_{u}, t>0\right\}$ and $U(\cdot, t) \in A$ for each $t>0$. Furthermore $U$ satisfies the heat equation:

$$
(\partial / \partial t-\Delta) U(x, t)=0 \quad \text { in } R_{+}^{n+1}
$$

For every $\varepsilon>0$ we have

$$
|U(x, t)| \leqq C_{\varepsilon} e^{\varepsilon / t} \quad \text { in } R_{+}^{n+1}
$$

We have for any $\delta>0$

$$
U(\cdot, t) \longrightarrow 0
$$


uniformly in $\left\{x \in R^{n} ; \operatorname{dis}(x, K) \geqq \delta\right\}$ as $t \rightarrow 0_{+}$; we have

$$
\begin{array}{r}
U(\cdot, t) \longrightarrow u \quad \text { in } A^{\prime}(K) \text { as } t \longrightarrow 0_{+}, \text {i.e. } \\
u(\phi)=\lim _{t \rightarrow 0_{+}} \int U(x, t) \chi(x) \phi(x) d x, \quad \phi \in A,
\end{array}
$$

for any $\chi \in C_{0}^{\infty}\left(R^{n}\right)$ such that $\chi=1$ in a neighborhood of $K$. Conversely, every $C^{\infty}$-function $U(x, t)$ defined in $R_{+}^{n+1}$ satisfying the conditions (1.6), (1.7) and (1.8) can be expressed in the form (1.5) with unique element $u \in$ $A^{\prime}(K)$.

Proof. Let $u \in A^{\prime}(K)$. Then we have obviously $U(x, t) \in C^{\infty}\left(R_{+}^{n+1}\right)$ and (1.6). We take $z=y+$ i $\eta$ and observe that

$$
R e-\left(x-y-i \gamma_{1}\right)^{2}=-(x-y)^{2}+\eta^{2},
$$

then by applying (1.4) we have (1.7) if we take $|\eta|$ sufficiently small. We have also (1.8) by the same argument. Now let

$$
G(y, t)=\int E(x-y, t) \chi(x) \phi(x) d x, \quad \phi \in A,
$$

where $\chi \in C_{0}^{\infty}\left(R^{n}\right)$ such that $\chi=1$ in a neighborhood of $K$. Then we have by Proposition 1.1

$$
G(\cdot, t) \longrightarrow \phi \text { in } A(K) \text { as } t \longrightarrow 0 .
$$

We have

$$
\int U(x, t) \chi(x) \phi(x) d x=u_{y}(G(y, t))
$$

by taking limit of the Riemann sum of the left hand side. By applying (1.11), we have (1.9)'. Now we will prove the converse. We shall use some fundamental theorems on ultradistributions, though we will not recall here basic definition about ultradistributions. We refer the reader to [5], [6] and [7], and will use notations of these papers such as $\mathscr{D}^{\{s}(\Omega)$, $\mathscr{E}^{\{s\}}(\Omega), \mathscr{D}^{\{s\}}(\Omega)$ and $\mathscr{E}^{\{s\}}(\Omega), s>1$. Let $U=U(x, t)$ be a function defined in $R_{+}^{n+1}$ satisfying (1.6), (1.7) and (1.8). Then by (1.7) we can extend $U$ beyond the hyperplane $t=0$ as an ultradistribution $\tilde{U} \in \mathscr{D}^{\{2\}}\left(R^{n+1}\right)$ such that

$$
\tilde{U}=\left\{\begin{array}{cc}
U(x, t) & t>0 \\
0 & t<0 .
\end{array}\right.
$$


This follows from the fundamental property of ultradistributions, (cf. [7], Theorem 2.27). We have $\tilde{U} \in C^{\infty}\left(R^{n+1} \backslash(K \times\{0\})\right)$ and

$$
(\partial / \partial t-\Delta) \tilde{U}(x, t)=f \quad \text { in } R^{n+1},
$$

where $f \in \mathscr{E}^{\mathfrak{R}{ }^{\{2\}} \prime}(K \times\{0\})$. Then $f$ is uniquely represented as

$$
f=\sum_{i=0}^{\infty} f_{k}(x) \otimes \delta^{(k)}(t)
$$

with $f_{k}(x) \in \mathscr{E}^{\{2\}}(K), k=0,1, \cdots$, satisfying the following conditions:

For every $L>0$ and $h>0$ there is a constant $C$ such that

$$
\left|f_{k}(\phi)\right| \leqq C L^{k} k !^{-2} \sup _{\substack{x \in K \\ \alpha}} \frac{\left|D^{\alpha} \phi(x)\right|}{h^{|\alpha|} \alpha !^{2}}, \quad \phi \in \mathscr{E}^{\left\{{ }^{22}\right.}\left(R^{n}\right)
$$

and

$$
\operatorname{supp} f=\overline{\cup \operatorname{supp} f_{k}} \times\{0\}, \quad \text { (cf. [6], Theorem 3.1) . }
$$

We define an ultradistribution $v \in \mathscr{D}^{\{2\}}\left(R^{n+1}\right)$ as follows:

$$
v(x, t)=\left\{\begin{array}{cc}
\sum_{k=0}^{\infty} \int\left(\partial_{t}\right)^{k} E(x-y, t) f_{k}(y) d y & t>0, \\
0 & t<0 .
\end{array}\right.
$$

Here the integrals in the right hand side are taken in the sen seof distributions. Namely, we have formally

$$
v(x, t)=\iint E(x-y, t-\tau) f(y, \tau) d y d \tau .
$$

In [10], we have shown that $E(x, t) \in \mathscr{D}^{\{2\} \prime}\left(R^{n+1}\right)$ and

$$
(\partial / \partial t-\Delta) E(x-y, t-\tau)=\delta(x-y, t-\tau)
$$

also in the ultradistribution sense. We have

$$
(\partial / \partial t-\Delta) v(x, t)=f \quad \text { in } R^{n+1} .
$$

We will show that $\tilde{U}=v$. First we have $\tilde{U}-v \in \mathscr{D}^{\{2\}}\left(R^{n+1}\right)$ and

$$
(\partial / \partial t-\Delta)(\tilde{U}-v)=0 \quad \text { in } R^{n+1} \text {. }
$$

Using the relation (1.16) and the well known fact that $E(x, t) \in \mathscr{E}_{x, t}^{\{1,2\}}\left(R^{n+1} \backslash\{0\}\right)$, or by Gevrey hypoellipticity for the heat equation proved in [10], we have

$$
\tilde{U}-v \in \mathscr{E}^{\{2\}}\left(R^{n+1}\right) \text {. }
$$


Furthermore, by applying (1.8) for $\tilde{U}$ and $v$ and by (1.18) we can easily show that there is a number $M>0$ such that

$$
|\tilde{U}(x, t)-v(x, t)| \leqq M, \quad t \geqq 0 .
$$

By the well known uniqueness theorem for the solutions of Cauchy problem of the heat equation we have $\tilde{U}-v=0$. It remains to prove that there exists an element $v_{0}$ of $A^{\prime}(K)$ such that

$$
v(\cdot, t) \longrightarrow v_{0} \text { in } A^{\prime}(K) \text { as } t \longrightarrow 0_{+} .
$$

For every $t>0$, we have

$$
v(x, t)=\sum_{k=0}^{\infty} \int \Delta^{k} E(x-y, t) f_{k}(y) d y .
$$

Again the integrals are taken in the sense of distributions. Let $\chi \in C_{0}^{\infty}\left(R^{n}\right)$ and $\chi=1$ in a neighborhood of $K$. We have for $\phi \in A$

$$
\begin{aligned}
& \int v(x, t) \chi(x) \phi(x) d x \\
& \quad=\sum_{k=0}^{\infty} \int\left(\int E(x-y, t) \Delta^{k}(\chi(x) \phi(x)) d x\right) f_{k}(y) d y .
\end{aligned}
$$

By using (1.13), we can show that the right hand side tends to

$$
\sum_{k=0}^{\infty} f_{k}\left(\Delta^{k} \phi\right) \equiv v_{0}(\phi)
$$

as $t \rightarrow 0_{+}$and we have $v_{0} \in A^{\prime}(K)$. This completes the proof.

We set

$$
A^{\prime}\left(R^{n}\right)=\bigcup_{K \in R^{n}} A^{\prime}(K) .
$$

TheORem 1.3 (cf. [3], Theorem 9.1.6). If $u \in A^{\prime}\left(R^{n}\right)$ then there is a smallest compact set $K \subset R^{n}$ such that $u \in A^{\prime}(K)$; it is called the support of $u$.

Proof. Let $K$ be the intersection of all compact sets $K^{\prime}$ such that $u \in A^{\prime}\left(K^{\prime}\right)$. By Theorem 1.2 a function $U(x, t)$ is uniquely defined and satisfies the heat equation in $R^{n+1} \backslash(K \times\{0\})$. Noting that $u=\lim _{t \rightarrow 0+} U(\cdot, t)$, we have $u \in A^{\prime}(K)$.

THeOREM 1.4 (cf. [3], Theorem 9.1.8). If $K_{1}, \cdots, K_{r}$ are compact subsets of $R^{n}$ and $u \in A^{\prime}\left(K_{1} \cup \cdots \cup K_{r}\right)$, then one can find $u_{j} \in A^{\prime}\left(K_{j}\right)$ so that 


$$
u=u_{1}+\cdots+u_{r} .
$$

Proof. It is sufficient to prove the statement when $r=2$. Let $U(x, t)$ be the function defined by (1.5). The theorem will be proved if we can split into a sum

$$
U=U_{1}+U_{2},
$$

where $U_{j}$ satisfies the conditions (1.6), (1.7) and (1.8) with respect to $K_{\jmath}$. To do so we take the extention $\tilde{U} \in \mathscr{D}^{[2] \prime}\left(R^{n+1}\right)$ as in the proof of Theorem 1.2:

$$
\tilde{U}(x, t)=\left\{\begin{array}{cc}
U(x, t) & t>0, \\
0 & t<0 .
\end{array}\right.
$$

Then $\tilde{U}$ satisfies the heat equation outside $\tilde{K}_{1} \cup \tilde{K}_{2}$ where $\tilde{K}_{j}=K_{j} \times\{0\}$. We take a function $\psi \in C^{\infty}\left(R^{n+1} \backslash\left(\tilde{K}_{1} \cap \tilde{K}_{2}\right)\right)$ constructed in [3], Corollary 1.4.11 such that $\psi=0$ for large $|x|+|t|$ and near $\tilde{K}_{2} \backslash\left(\tilde{K}_{1} \cap \tilde{K}_{2}\right)$, $\psi=1$ neax $\tilde{K}_{1} \backslash\left(\tilde{K}_{1} \cap \tilde{K}_{2}\right)$ and $\psi \in L^{\infty}\left(R^{n+1}\right)$. Here "near" means in the sense of the slowly varying metric defined in [3], Chapter 1 . We will split $\tilde{U}$ as follows:

$$
\tilde{U}_{1}=\psi \tilde{U}-\tilde{V}, \quad \tilde{U}_{2}=(1-\psi) \tilde{U}+\tilde{V} .
$$

Here we define $\psi \tilde{U} \in \mathscr{E}^{\{2\}^{\prime}}\left(R^{n+1}\right)$ such that $\psi \tilde{U}=0$ near $\tilde{K}_{2} \backslash\left(\tilde{K}_{1} \cap \tilde{K}_{2}\right)$ and $(1-\psi) \tilde{U}=0$ near $\tilde{K}_{1} \backslash\left(\tilde{K}_{1} \cap \tilde{K}_{2}\right)$, which is well defined by Theorem 2.27 of [7]. We have

$$
(\partial / \partial t-\Delta)(\psi \tilde{U})=\tilde{F}+f,
$$

where $\tilde{F}$ and $f$ are in $\mathscr{E}^{\{2\}^{\prime}}\left(R^{n+1}\right)$ such that

$$
\tilde{F}=\left\{\begin{array}{cc}
(\partial / \partial t-\Delta)(\psi U) & t>0 \\
0 & t<0
\end{array}\right.
$$

and $f \in \mathscr{E}^{[2] \prime}\left(R^{n+1}\right)$, supp $f \subset \tilde{K}_{1}$. Now we define

$$
\tilde{V}(x, t)=E * \tilde{F}(x, t) \in \mathscr{D}^{\{2\}}\left(R^{n+1}\right)
$$

and $V(x, t)=\tilde{V}(x, t)$ for $t>0$. Then we have $\tilde{V} \in C^{\infty}\left(R^{n+1} \backslash \tilde{K}_{1} \cap \tilde{K}_{2}\right)$, supp $\tilde{V} \subset R_{+}^{n+1}$ and $V(\cdot, t) \rightarrow 0$ uniformly in $\left\{x\right.$; dis $\left.\left(x, K_{1} \cap K_{2}\right) \geqq \delta\right\}$ for any $\delta>0$ as $t \rightarrow 0_{+}$. Here we use the pseudolocal property of $E *$ as a pseudodifferential operator, (cf. [1] and [10]). Since we have

$$
V(x, t)=\psi U-E * f(x, t), \quad t>0,
$$

we have 


$$
V(x, t)=O\left(e^{\varepsilon / t}\right) \quad t \longrightarrow 0_{+}
$$

for any $\varepsilon>0$. Thus we have the desired property for $U_{1}=\psi U-V$ and $U_{2}=(1-\psi) U+V$ for $t>0$.

We define the general hyperfunctions following [3].

Definition 1.3. If $\Omega \subset R^{n}$ is open and bounded we define the space of hyperfunctions $B(\Omega)$ by

$$
B(\Omega)=A^{\prime}(\bar{\Omega}) / A^{\prime}(\partial \Omega) .
$$

Definition 1.4. Ler $u \in A^{\prime}(\bar{\Omega})$ then the support of the class $u$ of $u$ in $B(\Omega)$ is defined by $\operatorname{supp} u^{\circ}=\Omega \cap \operatorname{supp} u$.

Here we omit the precise argument on general hyperfunctions on $R^{n}$ and we refer the reader to [3], Section 8.2.

Remark 1.1. If $u \in \mathscr{E}^{\prime}\left(R^{n}\right)$ then $u$ defines an element in $A^{\prime}\left(R^{n}\right)$ with the same support. In fact, the function $U(x, t)$ defined by (1.5) has the $\mathscr{D}^{\prime}$ limit $u$ as $t \rightarrow 0_{+}$and the condition (1.7) in Theorem 1.2 is replaced by

$$
|U(x, t)| \leqq C_{N} t^{-N}, \quad t>0,
$$

for some integer $N \geqq 0$.

Remark 1.2. If $u \in \mathscr{E}^{\{s\} '}\left(R^{n}\right), s>1$, then $u$ also defines an element in $A^{\prime}\left(R^{n}\right)$ with the same support. We can see that the condition (1.7) in Theorem 1.2 is replaced by

$$
|U(x, t)| \leqq C_{\varepsilon} \exp \left(\varepsilon / t^{1 / 2 s-1}\right), \quad t>0,
$$

for any $\varepsilon>0$. This will be proved in a forthcoming paper.

Thus we have the characterization of Schwartz and ultradistributions at the same time in such a way and we have the injections preserving supports

$$
\mathscr{E}^{\prime}\left(R^{n}\right) \smile \mathscr{E}^{[s\}}\left(R^{n}\right) \smile A^{\prime}\left(R^{n}\right)
$$

for any $s, 1<s<\infty$.

\section{§2. The Fourier-Laplace transformation in $A^{\prime}\left(R^{n}\right)$}

As an application of Theorem 1.2 we shall give a simple proof of Paley-Wiener-Schwartz theorem for hyperfunctions. The following method can be applied in each case of $\mathscr{D}\left(R^{n}\right), \mathscr{E}^{\prime}\left(R^{n}\right), \mathscr{E}^{\{s\}}\left(R^{n}\right), 0<s<\infty$, etc. 
Let $u \in A^{\prime}(K), K$ compact in $R^{n}$, then the Fourier-Laplace transform

$$
\hat{u}(\zeta)=u(\exp (-i\langle\cdot, \zeta\rangle))
$$

is an entire function such that for every $\varepsilon>0$

$$
|\hat{u}(\zeta)| \leqq C_{\varepsilon} \exp (L|\eta|+\varepsilon|\xi|), \quad \zeta=\xi+i \eta \in C^{n},
$$

where $L=\sup _{x \in K}|x|$. This follows from (1.4), (or (1.3)).

Theorem 2.1. If $F(\zeta)$ is an entire analytic function satisfying the estimates (2.1) with constant $L \geqq 0$. Then $F(\zeta)$ is the Fourier-Laplace transform of a unique element in $A^{\prime}\left(S_{L}\right)$, where $S_{L}=\left\{x \in R^{n} ;|x| \leqq L\right\}$.

Proof. We define a function $\hat{U}(\zeta, t)$ as follows:

$$
\hat{U}(\zeta, t)=F(\zeta) \exp \left(-t \zeta^{2}\right), \quad t>0 .
$$

Then we have $\hat{U}(\xi, t)=F(\zeta) \exp \left(-t|\xi|^{2}\right) \in \mathscr{S}\left(R_{\tilde{\xi}}^{n}\right)$ for every $t>0$. Let

$$
U(x, t)=(2 \pi)^{-n} \int e^{i\langle x, \xi\rangle} \hat{U}(\xi, t) d \xi, \quad t>0 .
$$

Then $U(x, t) \in C^{\infty}\left(R_{+}^{n+1}\right)$ and satisfies

$$
(\partial / \partial t-\Delta) U(x, t)=0 \quad \text { in } R_{+}^{n+1} .
$$

By (2.1) we have for every $\varepsilon>0$

$$
\begin{aligned}
|U(x, t)| & \leqq C_{\varepsilon} \int e^{|\xi|(\varepsilon-t|\xi|} d \xi \\
& =C_{\varepsilon} \int_{|\xi| \leqq \varepsilon / t}+C_{\varepsilon} \int_{|\xi| \geqq \varepsilon / t} \\
& \equiv I_{1}+I_{2} .
\end{aligned}
$$

We have

$$
I_{1} \leqq C_{\varepsilon} \int_{|\xi| \leqq \varepsilon / t} \exp \left(\varepsilon^{2} \mid t\right) d \xi \leqq C_{\varepsilon}^{\prime} \exp (\varepsilon / t), \quad t>0
$$

and

$$
\begin{aligned}
I_{2} \leqq & \left.C_{\varepsilon} \int_{|\xi| \geqq \varepsilon / t} \exp \left(-\varepsilon\left|t^{\prime} t\right| \xi \mid-\varepsilon\right)\right) d \xi \\
& =C_{\varepsilon} \exp \left(\varepsilon^{2} / t\right) \iint_{|\xi| \leqq \varepsilon / t} e^{-\varepsilon|\xi|} d \xi \leqq C_{\varepsilon}^{\prime \prime} \exp (\varepsilon / t), \quad t>0 .
\end{aligned}
$$

Thus we have for every $\varepsilon>0$ the estimate 


$$
|U(x, t)| \leqq C_{\varepsilon} \exp (\varepsilon / t) \quad \text { in } R_{+}^{n+1} .
$$

Now shifting the integration in (2.3) into the complex domain, we have

$$
U(x, t)=(2 \pi)^{-n} \int e^{i\langle x, \xi+i \eta\rangle} \hat{U}(\xi+i \eta, t) d \xi
$$

for an arbitrary fixed vector $\eta \in R^{n}$. Estimating the integral by using (2.1), we have

$$
\begin{aligned}
|U(x, t)| & \leqq C_{\varepsilon} \exp \left(L|\eta|-x \eta+t \eta^{2}\right) \int \exp (|\xi|(\varepsilon-t|\xi|)) d \xi \\
& \leqq C_{\varepsilon}^{\prime \prime} \exp \left(L|\eta|-x \eta+t \eta^{2}+\varepsilon / t\right) .
\end{aligned}
$$

We choose $\eta=x /(\sqrt{t}|x|)$ for $x \in R^{n} \backslash S_{L+\delta}$ and $\varepsilon=\delta / 2, \delta>0$. Then we have

$$
|U(x, t)| \leqq C_{\varepsilon}^{\prime \prime} \exp (-\delta / 2 \sqrt{t}+1), \quad t>0 .
$$

Hence by Theorem 1.2 there exists $u \in A^{\prime}\left(S_{L}\right)$ such that $U(\cdot, t) \rightarrow u$ in $A^{\prime}\left(S_{L}\right)$ in the sense of (1.9). We have

$$
\int U(x, t) e^{-i\langle x, \xi\rangle} d x \longrightarrow u\left(e^{-i\langle x, \xi\rangle}\right)=\hat{u}(\xi) \quad \text { as } t \longrightarrow 0_{+}
$$

and

$$
\int U(x, t) e^{-i\langle x, \xi\rangle} d x=F(\xi) e^{-t|\xi|^{2}} \longrightarrow F(\xi) \quad \text { as } t \longrightarrow 0_{+} .
$$

Thus we have $F(\xi)=\hat{u}(\xi)$. The uniqueness follows from the fact that

$$
u(P)=P(-D) \hat{u}(0)=P(-D) F(0)
$$

for every polynomial $P$.

Q.E.D.

\section{$\S 3$. Hypoellipticity of pseudodifferential equations in hyperfunction spaces}

We recall first a symbol class of analytic pseudodifferential operators considered in [1], [9], [10] and [12].

Definition 1.1. Let $-\infty<m<\infty$. We denote by $S_{1,0,1}^{m}\left(\Omega \times R^{n}\right)$ the set of all $a(x, \xi) \in C^{\infty}\left(\Omega \times R^{n}\right)$ such that for every compact set $K$ of $\Omega$ there are positive constants $C_{0}, C_{1}$ and $B$ such that

$$
\sup _{x \in K}\left|a_{(\beta)}^{(\alpha)}(x, \xi)\right| \leqq C_{0} C_{1}^{|\alpha+\beta|} \alpha ! \beta !(1+|\xi|)^{m-|\alpha|}, \quad|\xi| \geqq B|\alpha|,
$$


where

$$
a_{(\beta)}^{(\alpha)}(x, \xi)=\partial_{\xi}^{\alpha} D_{x}^{\beta} a(x, \xi) .
$$

We associate with such a symbol $a(x, \xi)$ a pseudodifferential operator $a(x, D)$ as usual:

$$
a(x, D) u(x)=(2 \pi)^{-n} \iint e^{i\langle x-y, \xi\rangle} a(x, \xi) u(y) d y d \xi, \quad u \in \mathscr{D}(\Omega) .
$$

It is well known that $a(x, D)$ is a linear continuous form from $\mathscr{D}(\Omega)$ into $\mathscr{E}(\Omega)$, and it is extended to a continuous mapping from $\mathscr{E}^{\prime}(\Omega)$ into $\mathscr{D}^{\prime}(\Omega)$ (cf. [2]). We define the transpose of $a(x, D)$ as follows:

$$
{ }^{t} a(x, D) v(y)=(2 \pi)^{-n} \iint e^{i\langle x-y, \xi\rangle} a(x, \xi) v(x) d x d \xi, \quad v \in \mathscr{D}(\Omega) .
$$

Then we have

$$
\langle a(x, D) u(x), v(x)\rangle=\left\langle u(x),{ }^{t} a(x, D) v(x)\right\rangle, \quad u, v \in \mathscr{D}(\Omega) .
$$

Furthermore as a special case of the results of [9] and [10], we know that $a(x, D)$ and ${ }^{t} a(x, D)$ are extended to continuous mappings from $\mathscr{E}^{\{s\}}(\Omega)$ into $\mathscr{D}^{\{s\}}(\Omega)$ for every $s, 1<s<\infty$.

Now let $u \in A^{\prime}(K), K$ compact in $\Omega$. Take a function $\chi \in C_{0}^{\infty}(\Omega)$ such that $\chi=1$ in a neighborhood of $K$. Let $U(x, t)$ be the function defined by (1.15):

$$
U(x, t)=u_{y}(E(x-y, t)), \quad t>0 .
$$

Then for every $\phi \in A$, we have

$$
\int \chi a(x, D) \chi U(x, t) \phi(x) d x=\int \chi(x) U(x, t)^{t} a(x, D)\left(\chi_{\phi}\right)(x) d x, \quad t>0 .
$$

By virtue of the analytic pseudolocal property of ${ }^{t} a(x, D)$ proved in [1], we have ${ }^{t} a(x, D)(\chi \phi)(x) \in A(K)$ and the right hand side tends to

$$
\left\langle u,{ }^{t} a(x, D)\left(\chi_{\phi}\right)(x)\right\rangle
$$

as $t \rightarrow 0$, (cf. $\left.(1.9)^{\prime}\right)$. We define $\chi a(X, D) u$ by the formula

$$
\langle\chi a(x, D) u, \phi\rangle=\left\langle u,{ }^{t} a(x, D) \chi \phi\right\rangle, \quad \phi \in A .
$$

Then we have $\chi a(x, D) u \in A^{\prime}(\operatorname{supp} \chi)$. On the other hand we have

$$
(1-\chi) a(x, D) u(x)=\int(1-\chi(x)) K(x, y) u(y) d y \in C^{\infty}(\Omega)
$$


where $K(x, y)$ is the kernel of $a(x, D)$ which is real analytic in $\Omega \times \Omega \backslash \Delta$, $\Delta=\{(x, x) ; x \in \Omega\}$, (cf. [1]). We define

$$
a(x, D) u \equiv \chi a(x, D) u+(1-\chi) a(x, D) u \in B(\Omega) .
$$

We can easily see that this definition is independent of such a function $\chi$ and agrees with the usual definition of $a(x, D) u \in \mathscr{D}^{\prime}(\Omega)$ when $u \in \mathscr{E}^{\prime}(\Omega)$.

THEOREm 3.1. The analytic (and $C^{\infty}$ ) pseudolocal property holds for $a(x, D)$ in $\Omega$. Namely, if $u \in A^{\prime}(K), K$ compact in $\Omega$, is real analytic $\left(C^{\infty}\right)$ in some open set $\omega \subset K$, then $a(x, D) u$ is also real analytic $\left(C^{\infty}\right)$ in $\omega$.

Proof. Take an arbitrary open set $\omega_{1} \subset \omega$ such that $\bar{\omega}_{1} \subset \omega$ and devide $u$ so that

$$
u=u_{1}+u_{2}, \quad \operatorname{supp} u_{1} \subset \bar{\omega}_{1}, \quad \operatorname{supp} u_{2} \subset K \backslash \omega_{1} .
$$

(cf. Theorem 1.4.)

Then obviously $u_{1} \in \mathscr{E}^{\prime}\left(\bar{\omega}_{1}\right)$ and $u_{1}$ is analytic in $\omega_{1}$. By the analytic pseudolocal property of $a(x, D)$ in the sense of Schwartz distributions proved in [1], we see $a(x, D) u_{1}$ is analytic in $\omega_{1}$. On the other hand we have

$$
\left.a(x, D) u_{2}\right|_{\omega_{1}}=\int K(x, y) u_{2}(y) d y, \quad x \in \omega_{1}
$$

and this is also analytic in $\omega_{1}$, where $K(x, y)$ is the kernel of $a(x, D)$. Summing up, we see $a(x, D) u$ is analytic in $\omega_{1}$. Hence $a(x, D) u$ is analytic in $\omega$. The proof in $C^{\infty}$ case is obtained similarly.

As a supplement of the results of [10], Theorem 2.2 and [9], Theorem 3.1 we shall give the following theorem.

TheOREM 3.2. Let $a(x, \xi) \in S_{1,0,1}^{m}\left(\Omega \times R^{n}\right)$ and assume that there are positive constants $c$ and $B$ and $-\infty<m^{\prime}<\infty$ such that

$$
|a(x, \xi)| \geqq c|\xi|^{m^{\prime}}, \quad x \in \Omega, \quad|\xi| \geqq B .
$$

Also assume that for any compact set $K \subset \Omega$, there are positive constants $C_{0}$ and $C_{1}$ such that

$$
\left|a_{(\beta)}^{(\alpha)}(x, \xi)\right| \leqq C_{0} C_{1}^{|\alpha+\beta|} \alpha ! \beta !|a(x, \xi)||\xi|^{-|\alpha|}, \quad x \in K, \quad|\xi| \geqq B|\alpha| .
$$

Then if $u \in A^{\prime}(K), K$ compact of $\Omega$, and $a(x, D) u$ is real analytic $\left(C^{\infty}\right)$ in an open set $\omega \subset K$, we see $u$ is also real analytic $\left(C^{\infty}\right)$ in the same set $\omega$. 
Proof. Let $u \in A^{\prime}(K)$ and $a(x, D) u$ is analytic in $\omega, \omega \subset K$. Take a function $h \in C_{0}^{\infty}(\Omega)$ such that $h=1$ in a neighborhood of $K$. Then $h a(x, D) u$ is also analytic in $\omega$. In [1], we have proved that there is a parametrix $b(x, D)$ of $a(x, D)$ such that it's smybol $b(x, \xi) \in S_{1,0,1}^{-m^{\prime}}\left(\Omega \times R^{n}\right)$ and

$$
b(x, D) h a(x, D)=I+R \text { in } \Omega^{\prime}, \quad K \subset \Omega^{\prime} \subset \Omega,
$$

where $R$ is an integral operator with kernel $R(x, y)$ which is analytic in $\Omega^{\prime} \times \Omega$; (cf. [11]). The equality (3.6) also holds in the hyperfunction sense. In fact, choose $\chi \in C_{0}^{\infty}(\Omega)$ such that $\chi=1$ in a neighborhood $\Omega^{\prime}$ of $K$ and $\operatorname{supp} \chi \subset\{x ; h(x)=1\}$. Then by [1], Theorem 3.1, we have

$$
b(x, D) h a(x, D) \chi U(x, t)=\chi U(x, t)+\int R(x, y) \chi(y) U(y, t) d y,
$$

where $U(x, t)=u_{y}(E(x-y, t)) t>0$. Taking the limit as $t \rightarrow 0_{+}$, we see

$$
b(x, D) h a(x, D) u=u+\int R(x, y) u(y) d y \quad \text { in } \Omega^{\prime} .
$$

On the other hand, by Theorem 3.1 we have the left hand side is real analytic in $\omega$. Hence we have $u$ is analytic in $\omega$. This completes the proof.

\section{REFERENCES}

[1] S. Hashimoto, T. Matsuzawa et Y. Morimoto, Opérateurs pseudodifférentiels et classes de Gevrey, Comm. Partial Differential Equations, 8(12) (1983), 1277-1289.

[2] L. Hörmander, Pseudodifferential operators and hypoelliptic equations, Proc. Symp. Pure Math., 83 (1966), 129-209.

[3] - The analysis of linear partial differential operators, I, Springer-Verlag, Berlin Heidelberg New York Tokyo, 1983.

[ 4 ] M. Kashiwara, Introduction to the theory of hyperfunctions, In Sem. on microlocal analysis, Princeton Univ. Press, Princeton, N. J., 1979, 3-38.

[ 5 ] H. Komatsu, Ultradistributions, I; Structure theorems and a characterization, J. Fac. Sci. Univ. Tokyo, Sect. IA, 20 (1973), 25-105.

[6] - Ultradistributions, II; The kernel theorem and ultradistributions with support in a submanifold, J. Fac. Sci. Univ. Tokyo, Sect. IA, 24 (1977), 607-628.

[ 7] - Introduction to the theory of distributions (in Japanese), Iwanami Shoten, 1978.

[8] A. Martineau, Les hyperfonctions de M. Sato, Sém. Bourbaki 1960-1961, Exposé No. 214.

[9] T. Matsuzawa, Gevrey hypoellipticity of a class of pseudodifferential operators, to appear in Tôhoku Math. J.

[10] — Hypoellipticity in ultradistribution spaces, to appear in J. Fac. Sci. Univ. Tokyo. 
[11] S. Mizohata, On asymptotic expressions of symbols and formal symbols, Lecture Note at Kyoto Univ., 1985.

[12] F. Treves, Introduction to pseudodifferential and Fourier integral operators, I, Plenum Press, 1981.

Department of Mathematics

Faculty of Science

Nagoya University

Chikusa-ku, Nagoya 464

Japan 